\title{
Holographic QCD in the Veneziano Limit at a Finite Magnetic Field and Chemical Potential
}

\author{
Umut Gürsoy, ${ }^{1}$ Matti Järvinen, ${ }^{2}$ and Govert Nijs ${ }^{1}$ \\ ${ }^{1}$ Institute for Theoretical Physics and Center for Extreme Matter and Emergent Phenomena, Utrecht University, \\ Leuvenlaan 4, 3584 CE Utrecht, Netherlands \\ ${ }^{2}$ Institut de Physique Théorique Philippe Meyer and Laboratoire de Physique Théorique, École Normale Supérieure, \\ PSL Research University, CNRS, 24 rue Lhomond, 75231 Paris Cedex 05, France
}

(Received 22 September 2017; revised manuscript received 28 April 2018; published 11 June 2018)

\begin{abstract}
We investigate QCD-like gauge theories at strong coupling at a finite magnetic field $B$, temperature $T$, and quark chemical potential $\mu$ using the improved holographic QCD model, including the full backreaction of the quarks in the plasma. In addition to the phase diagram, we study the behavior of the quark condensate as a function of $T, B$, and $\mu$ and discuss the fate of (inverse) magnetic catalysis at a finite $\mu$. In particular, we observe that inverse magnetic catalysis exists only for small values of the chemical potential. The speed of sound in this holographic quark-gluon plasma exhibits interesting dependence on the thermodynamic parameters.
\end{abstract}

DOI: 10.1103/PhysRevLett.120.242002

Introduction.-Despite being the established theory of the strong force, the phase diagram of quantum chromodynamics is still largely unknown, especially at a finite chemical potential $\mu$ and in the presence of an external magnetic field $B$. Understanding every corner of this phase diagram, especially when the coupling is strong, is crucial not only for high energy physics but also for astrophysics and cosmology. For example, the quark-gluon plasma produced in heavy ion collisions, which is a strongly coupled quantum fluid produced along with large magnetic fields [1-7], is also the fabric of the core of neutron stars and magnetars, and the early Universe.

Strong coupling obstructs perturbative analysis and necessitates lattice QCD studies. Indeed, lattice QCD has been an extremely fruitful method in the study of the phase diagram at a finite magnetic field [8] and led to the recent discovery of inverse magnetic catalysis [9-12], in addition to confirmation of predicted phenomena such as magnetic catalysis [13-15]. However, this method is not fully functional [16] at a finite $\mu$ due to the notorious sign problem [17]. This has prompted an investigation of the QCD phase diagram at a finite chemical potential using alternative nonperturbative methods, such as effective field theories $[7,18]$ and holographic correspondence [19].

In this Letter, we take the first step in exploring this phase diagram at strong coupling using a full-fledged

Published by the American Physical Society under the terms of the Creative Commons Attribution 4.0 International license. Further distribution of this work must maintain attribution to the author(s) and the published article's title, journal citation, and DOI. Funded by SCOAP. holographic model for QCD at a finite temperature $T, \mu$, and $B$. We construct the holographic model as a bottom-up effective model that captures the qualitative salient features of QCD, such as asymptotic freedom, linear confinement, a discrete spectrum of hadrons and glueballs, and the phenomenon of inverse magnetic catalysis. Even though the choice of potentials involves some arbitrariness, the asymptotic IR behavior is fixed by these requirements [20] and our qualitative results in the IR should be applicable to any theory in the same universality class as QCD, as discussed at length in Ref. [21]. The numerical detailsespecially in the UV-may differ from those in QCD. To maintain all of these universal features relevant to QCD, we base our model on Refs. [20,22,23] extended to a finite chemical potential and magnetic field. In this extension it is crucial to capture the dynamics of quarks, and especially the quark condensate. As holographic models require a large number of colors $N_{c}$, to maintain a non-negligible influence of $B$ and $\mu$ on the quark sector [24,25] in this limit, one also needs to consider a large number of quark flavors $N_{f}$, arriving at the so-called Veneziano limit [26]:

$N_{c} \rightarrow \infty, \quad N_{f} \rightarrow \infty, \quad x \equiv N_{f} / N_{c}=$ const.

Thus, the holographic analog of QCD with two or three quark flavors would correspond to taking $x=2 / 3$ or 1 . Constructing a realistic holographic theory in the Veneziano limit is quite nontrivial, and important steps in this direction were taken in Refs. [27-30]. Subsequently, a uniform external magnetic field in this model was introduced in Refs. [31,32]. In this Letter, in addition to a finite $T$ and $B$, we extend the aforementioned model with a finite chemical potential. A central focus in our 
exploration is inverse magnetic catalysis [8], that is, the weakening of the quark condensate - and consequently a decrease in the chiral transition temperature $T_{\chi}$-with an increasing value of $B$. We stress that, as has been demonstrated on the lattice [33], backreaction of quarks is essential in order to capture the dynamics of the inverse catalysis. This observation is also supported by the earlier holographic studies [32,34-37]. A particularly pressing question is the fate of this phenomenon at a finite $\mu$.

Holographic QCD model.-We model the system of strongly coupled quarks and gluons in the Veneziano limit (1) by a "bottom-up" holographic model of QCD. Thus, the five-dimensional gravitational action contains two parts, corresponding to the two sectors, glue and flavor:

$$
S=S_{g}\left[g_{\mu \nu}, \phi\right]+x S_{f}\left[g_{\mu \nu}, \phi, \tau, L_{\mu}^{a}, R_{\mu}^{a}, V_{\mu}\right],
$$

where $x$ is the flavor to color ratio defined in Eq. (1), which we fix to 1 in this Letter. The gravitational action contains a bulk field corresponding to each marginal or relevant operator of QCD up to spin 2. These are the metric for the stress tensor, the dilaton for the scalar glueball operator $\operatorname{tr} G^{2}$, a complex scalar $\tau$ for the quark condensate $\langle\bar{q} q\rangle$, and non-Abelian gauge fields $L_{\mu}^{a}, R_{\mu}^{a}$, and $V_{\mu}$ for the left and right chiral currents conserved under the symmetry $\mathrm{SU}\left(N_{f}\right)_{L} \times \mathrm{SU}\left(N_{f}\right)_{R}$ and the baryon number $\mathrm{U}(1)_{B}$. We introduce [38] the (quark) chemical potential $\mu$ and a uniform magnetic field $B$ in the $x_{3}$ direction through the bulk gauge field dual to this baryon number:

$$
V_{\mu}=\left(\Phi(z),-x_{2} B / 2, x_{1} B / 2,0,0\right),
$$

where $z$ is the holographic direction, and the boundary value of the scalar potential gives the chemical potential $\mu=\Phi(0)$ [30,32]. The actions $S_{g}$ and $S_{f}$ above are otherwise the same as in Refs. [29,30] but with the potential parameter $c$ defined in Ref. [32] fixed to $c=0.4$ (instead of $c=1$ ) [39]. The model contains an energy scale $\Lambda \approx$ $1 \mathrm{GeV}$ that corresponds to the dynamically generated energy scale of QCD, which appears as an integration constant in the equations of motion. We use this integration constant to define the dimensionless combinations $T / \Lambda$, $\mu / \Lambda$, and $B / \Lambda^{2}$.

Phase diagram and sound speed.-The gravitational solutions satisfy the first law of thermodynamics, $d F=-s d T-n d \mu-M d B$, where $s, n$, and $M$ are the entropy, quark number density, and magnetization. In practice, we calculate the free energy by first evaluating, $s, n$, and $M$-which is easier for a dual black-hole solution - and then using the first law to integrate. An interesting phase diagram (at zero quark mass) results from competition between the following phases, as shown in Fig. 1: (i) a horizonless geometry with a nontrivial profile for $\tau$, called the thermal gas, that corresponds to the chirally broken confined hadron gas, shown as green; (ii) a black-hole

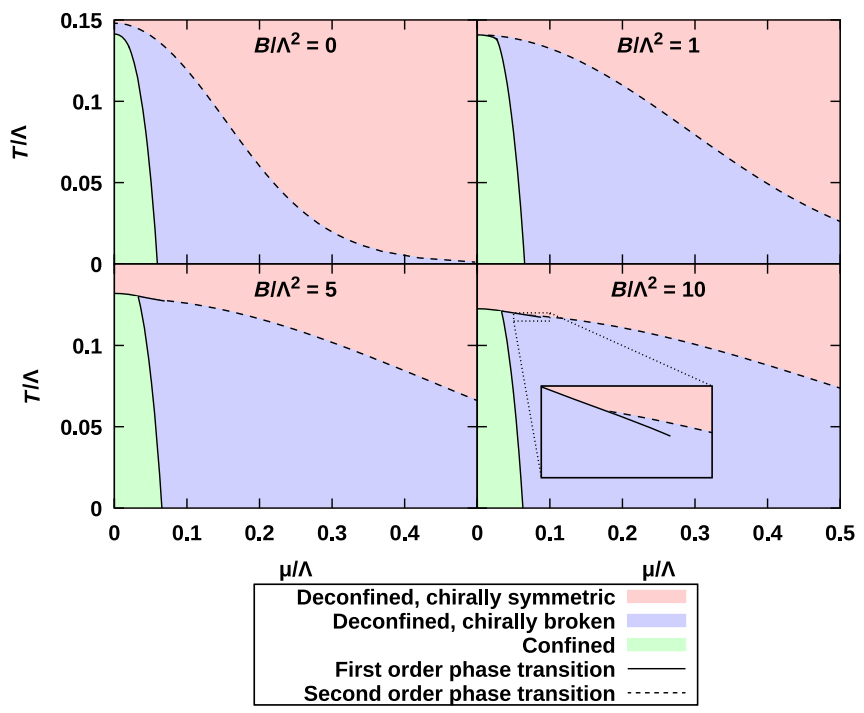

FIG. 1. The phase diagram on the $(\mu, T)$ plane at different values of the magnetic field.

solution with a nontrivial profile for $\tau$ that corresponds to a deconfined quark-gluon plasma where the chiral symmetry is broken, shown as blue; and (iii) a black-hole solution with a trivial $\tau$ that corresponds to a deconfined quarkgluon plasma with restored chiral symmetry, shown as pink [40].

We note that phase (ii), which appears only in a limited region of the phase diagram in the case $\mu=0$ of Ref. [32], extends into a sizable part of the phase space at $\mu>0$. Also, the first order deconfinement transition (between the green and blue regions in the figure) is affected little by $B$ for small values of $T$. Essentially, the effect of $B$ on the deconfinement transition is significant only when it merges with the chiral symmetry restoration for $B \gtrsim \Lambda^{2}$. The chiral symmetry restoration becomes first order between $0<$ $\mu / \Lambda \lesssim 0.1$ as $B$ grows. The first order line develops a second order end point and the second order transition line branches off the first order line, as shown in the inset of Fig. 1. Chiral symmetry is restored for a large $\mu$ at $T=0$ and a finite $B$ (i.e., on the horizontal axis at the right of the plotted region in Fig. 1), as expected from QCD. The free energy is independent of $T$ and $\mu$ in the confined phase [41], as we are working in the limit of a large value of $N_{c}$, which agrees with the "silver blaze" property of QCD at zero $T$ and a finite $\mu$ [42]. Finally, notice that baryons have not been implemented in the model, and their presence could potentially modify the phase diagram quantitatively at chemical potentials comparable to the baryon mass; that is to say, the phase separation curves would be modified.

Another thermodynamic observable that is very sensitive to the phase structure is the speed of sound, $c_{s}$. We study $c_{s}$ in the direction of the magnetic field, which can be computed by evaluating the derivative $-d F / d \epsilon$ while keeping $n / s$ and $B$ fixed. One obtains 


$$
c_{s}^{2}=\left.\frac{s d T+n d \mu}{T d s+\mu d n+B d M}\right|_{n / s, B} .
$$

The result is shown in Fig. 2 as a function of $T, \mu$, and $B$. We observe that $c_{s}$ exhibits a jump precisely at the first and second order phase boundaries in Fig. 1. We also find that it is enhanced both by $\mu$ and $B$ almost in the entire range of the parameter space, with the latter being in nontrivial agreement with lattice findings [43]. We also find that the conformal value of $c_{s}^{2}=1 / 3$ being crossed at various locations is somewhat unexpected from, but not in contradiction to, the findings of Refs. [44,45]. We checked that at large values of $T$-not visible in Fig. $2-c_{s}^{2}$ approaches the conformal value from below for all values of $\mu$ and $B$ considered, in accordance with Refs. [44,45].

Inverse magnetic catalysis.-As discussed in the Introduction, a pressing issue is the dependence of the quark condensate on the magnetic field at a finite chemical potential. One way to analyze this problem is to study the chiral transition temperature - the phase boundaries between the green or blue regions and the pink regions in Fig. 1 -as a function of $B$ and $\mu$ in more detail. In the Fig. 3 (top panel), we find that, for sufficiently small values of $\mu$, the chiral transition temperature in fact decreases with $B$ [46]. For larger values of $\mu$, the transition temperature increases with $B$. This behavior of the critical temperature suggests that inverse magnetic catalysis (weakening of the condensate with $B$ ) is found only near the critical temperature and at small chemical potentials. This can be confirmed by directly

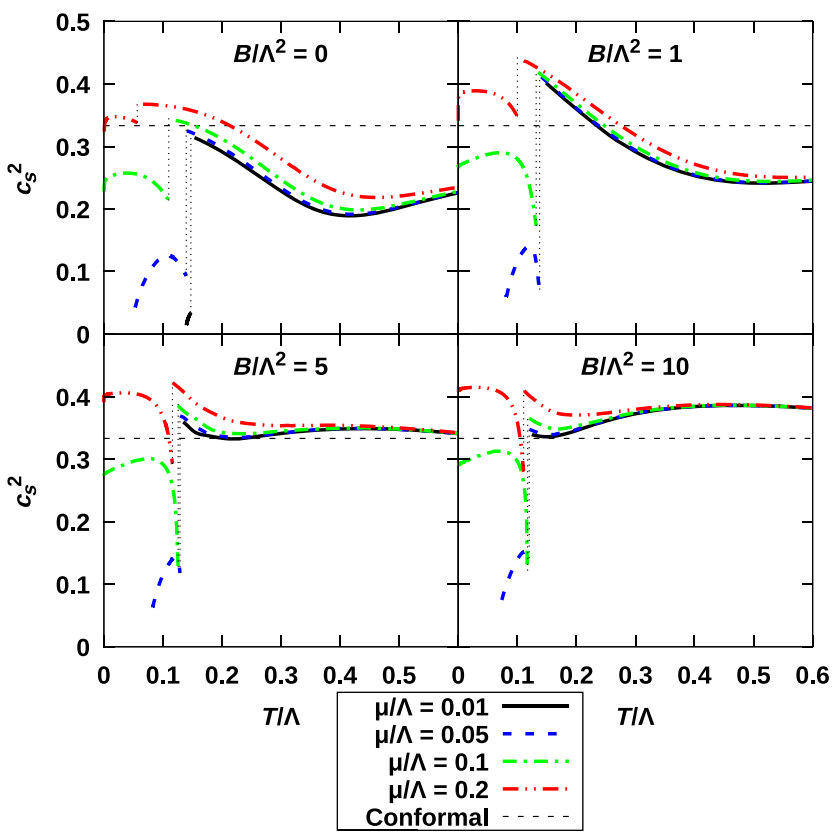

FIG. 2. The speed of sound squared $c_{s}^{2}$ as a function of temperature for different values of the magnetic field and the chemical potential. Numerical noise from these curves has been eliminated by using a high-momentum cutoff in Fourier space. evaluating the quark condensate in our holographic model. As explained in Ref. [32], the condensate can be read off from the near boundary asymptotics of the bulk complex scalar field $\tau$ that is dual to the quark condensate operator. Figure 3 (bottom panel) shows the regions of the phase space with inverse magnetic catalysis for small values of $B$. We conclude that increasing $\mu$ indeed makes it harder for inverse magnetic catalysis to occur, in general. Another observation from Fig. 3 (top panel) is that the interval in $\mu$, where $T_{\chi}$ decreases with $B$ and grows with increasing values of $B$. Therefore, the region where inverse catalysis is found, which is somewhat limited in the case of small values of $B$ shown in Fig. 3 (bottom panel), is expected to expand significantly as $B$ increases.

Normalizing the chiral condensate as

$$
\Sigma(T, \mu, B)=\frac{\langle\bar{q} q\rangle(T, \mu, B)}{\langle\bar{q} q\rangle(0,0,0)}
$$
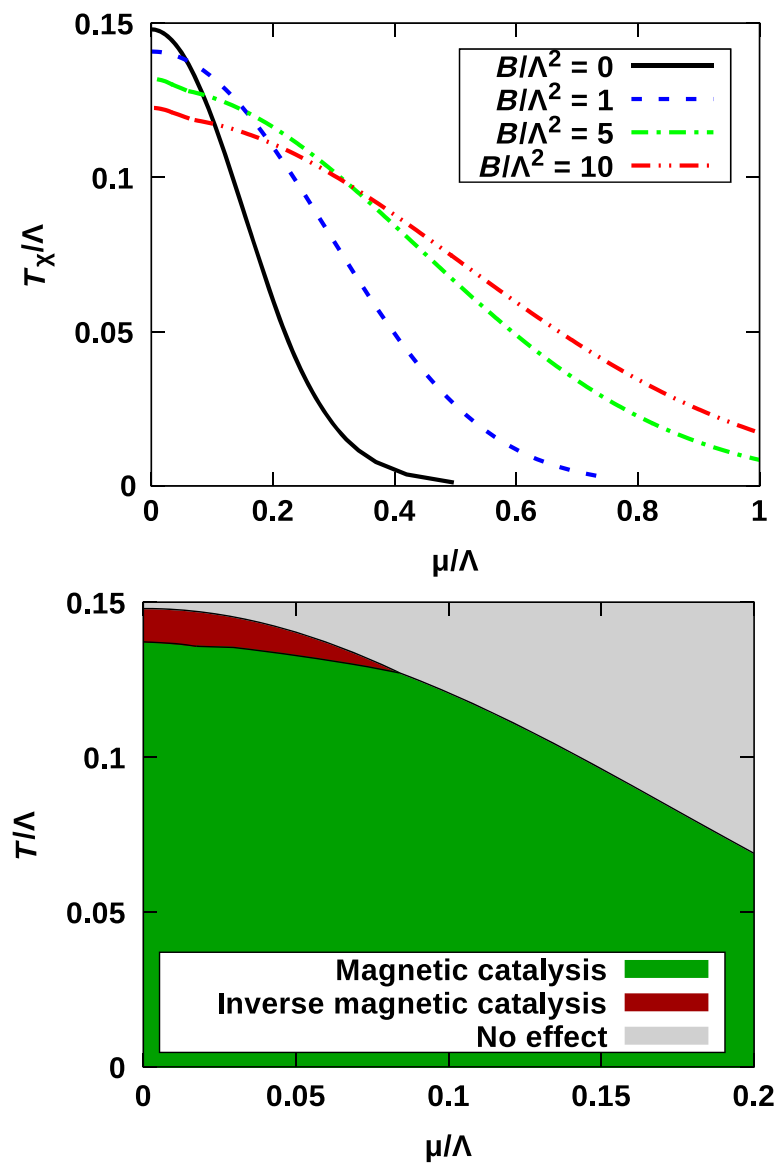

FIG. 3. (Top panel) The chiral transition temperature $T_{\chi}$ as a function of chemical potential for different values of the magnetic field. (Bottom panel) Region where (inverse) magnetic catalysis occurs for a small value of $B$, which is here defined by the sign of $\langle\bar{q} q\rangle_{B / \Lambda^{2}=0.1}-\langle\bar{q} q\rangle_{B / \Lambda^{2}=0}$. 


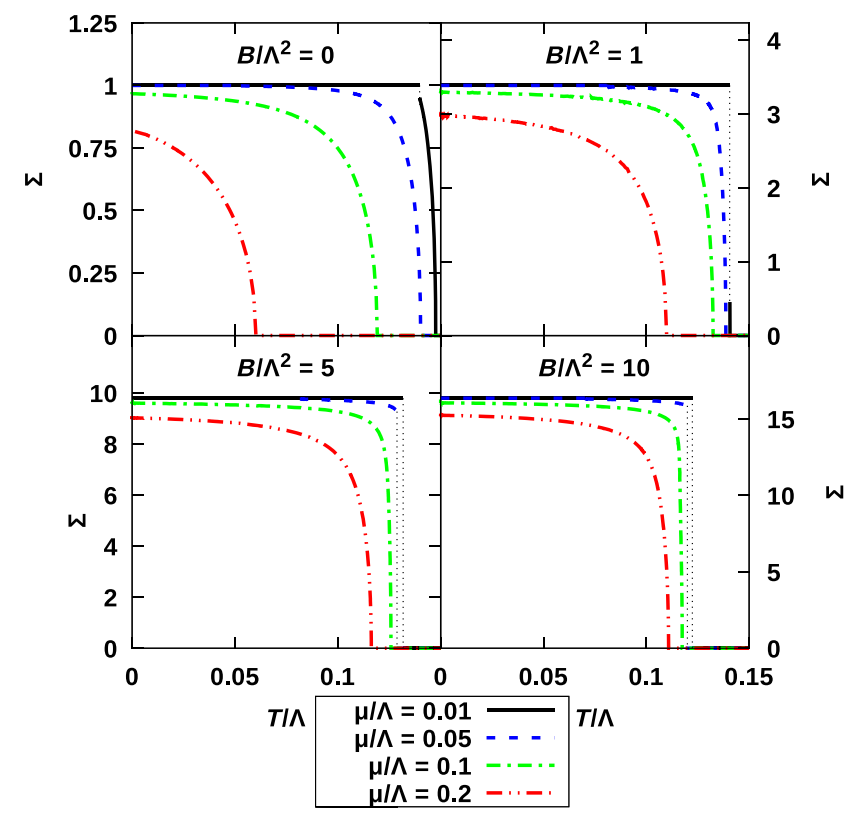

FIG. 4. The normalized chiral condensate $\Sigma$ as a function of temperature for different values of the magnetic field and the chemical potential.

we plot in Fig. 4 its dependence on $T$ for various choices of $\mu$ and $B$. We observe that $\Sigma$ always decreases with $T$, exhibiting discontinuities at the phase boundaries in Fig. 1. The reason for the absence of $T$ dependence of $\Sigma$ in the confined phase (the green phase in Fig. 1) is because this dependence is suppressed with $1 / N_{c}^{2}$ in our model in the large- $N$ limit $[32,41]$.

Finally, as observed in Refs. [32,47] and discussed in detail in the latter paper, magnetization can also be utilized to distinguish the magnetic and the inverse magnetic catalysis. In particular, whether the transition temperature increases or decreases with $B$ is correlated with whether the magnetization jumps up or down across a first order transition. Similarly, for a second order phase transition, e.g., the dashed curves in Fig. 1, one finds that

$$
\operatorname{sgn}\left(\frac{d T_{\chi}}{d B}\right)=\operatorname{sgn}\left(\frac{d M\left(T_{\chi}+\epsilon\right)}{d T}-\frac{d M\left(T_{\chi}-\epsilon\right)}{d T}\right)
$$

which follows from equating the entropies on a second order phase boundary. We observe in Fig. 5 that, for example, for small values of $\mu$ and $B$, one finds that $d T_{\chi} / d B<0$, an indication of inverse magnetic catalysis. In general, although the kinks in $M(T)$ are often too small to be visible in Fig. 5, the findings agree with those in Fig. 3.

Discussion.-There were two main results in our Letter. First was the phase diagram of a large- $N$ holographic QCD theory with full backreaction from the quark sector at a finite temperature, chemical potential, and magnetic field; see Fig. 1. We considered massless quarks and fixed the flavor to color ratio, Eq. (1), to be unity in this Letter.

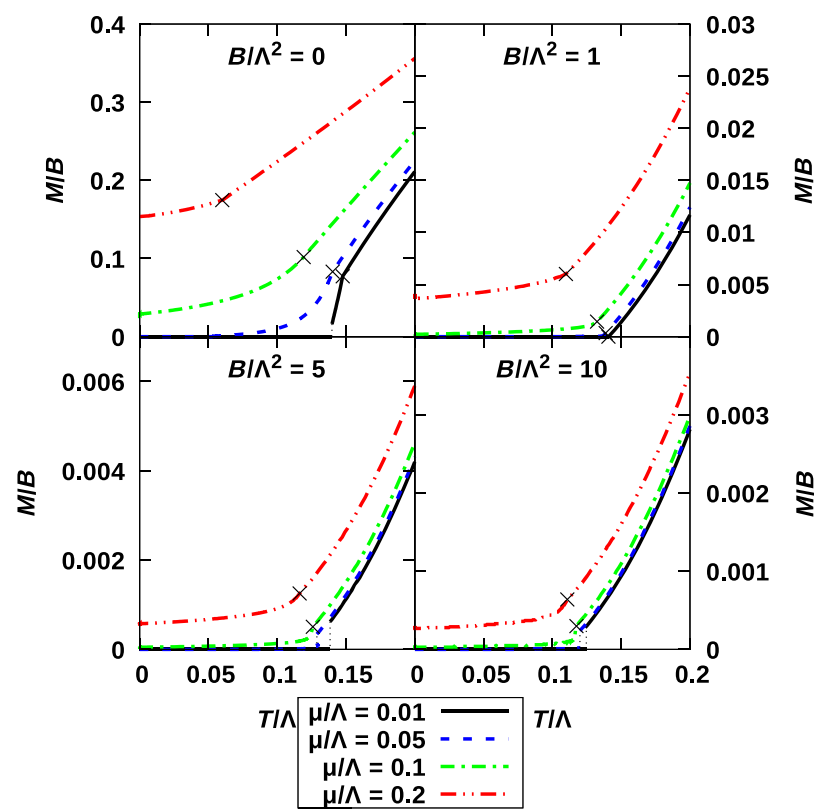

FIG. 5. The magnetization divided by the magnetic field strength as a function of $T, \mu$, and $B$. The $B=0$ result should be interpreted as the limit approaching $B=0$. The crosses denote the locations of the second order chiral transitions. Here, $M$ has been normalized so that $M_{T=0, \mu=0}=0$ for every value of $B$.

Generalization to massive quarks and the study of the phase diagram at different values of $x$ are two natural extensions. Also, we disregarded the possibility of inhomogeneous phases in this Letter. Whether they occur and compete with the phases here is an interesting question. We checked the thermodynamic stability of all of the phases shown in Fig. 1. It is remarkable that phase (ii) (the blue regions in Fig. 1), which is the deconfined plasma phase with broken chiral symmetry, seems to be a universal prediction of a variety of holographic models $[25,27,48-50]$. We found that this phase was also present at a finite $B$; moreover, it covered a larger part of the phase diagram for larger values of $B$.

Our second main result was how the region of inverse magnetic catalysis, which was observed on the lattice simulations [8] at vanishing values of $\mu$, extends to a finite $\mu$. There are differences in definition of this phenomenon in the literature. In our Letter, we followed the definition following the lattice findings [8], that is, we defined inverse magnetic catalysis as the weakening of the quark condensate, and, related to this, the decrease in the chiral transition temperature $T_{\chi}$ with increasing values of $B$. We observed both of these effects. The original work of Preis et al. [51] and the most recent follow-up [47], both of which use the Sakai-Sugimoto model [52], define the phenomenon as the decrease in the critical chemical potential with $B$ for small temperatures. Interestingly, the latter effect has also been seen in approximations to QCD which are directly based on field theory (see, e.g., Ref. [53]). 
Notice also that we observed inverse catalysis at relatively low values of $\mu$, where the sign problem is probably surmountable. Therefore, it may be possible to check our results on the lattice [54].

The choice of our model necessarily introduced a degree of arbitrariness in the particular values of thermodynamic observables. However, the main qualitative results, i.e., the generic phase diagram, the influence of $\mu$ and $B$ on the condensate and $c_{s}$, etc., are sensitive only to the asymptotic values of the potentials which are fixed by the physical requirements in QCD. The only exception seems to be inverse magnetic catalysis, which required, in our parametrization, taking the constant $c$ to be sufficiently small. A very important task ahead is to scan through different choices of potentials and to quantify the changes in the thermodynamic properties establishing the systematic errors of our effective model.

Our results were obtained by solving Einstein's equations numerically, which is necessarily complicated since it involves backreaction of the flavor branes. To facilitate our calculations and minimize the error and the time spent, we wrote a program in $\mathrm{C}++$, which was based in part on the earlier Mathematica code [55]. This program solves the equations of motion numerically using the LSODA algorithm, varying the boundary conditions in a grid to obtain data for the entire phase space.

We are grateful to G. Aarts, J. O. Andersen, A. BallonBayona, I. Iatrakis, A. Schmitt, and D. Zoakos for the discussions. This work was supported in part by the Netherlands Organisation for Scientific Research (NWO) under VIDI Grant No. 680-47-518, and the Delta Institute for Theoretical Physics (D-ITP), funded by the Dutch Ministry of Education, Culture and Science (OCW).

[1] D. E. Kharzeev, L. D. McLerran, and H. J. Warringa, Nucl. Phys. A803, 227 (2008).

[2] V. Skokov, A. Yu. Illarionov, and V. Toneev, Int. J. Mod. Phys. A 24, 5925 (2009).

[3] K. Tuchin, Phys. Rev. C 82, 034904 (2010); 83, 039903(E) (2011).

[4] L. McLerran and V. Skokov, Nucl. Phys. A929, 184 (2014).

[5] T. Vachaspati, Phys. Lett. B 265, 258 (1991).

[6] H. Tashiro, T. Vachaspati, and A. Vilenkin, Phys. Rev. D 86, 105033 (2012).

[7] V. A. Miransky and I. A. Shovkovy, Phys. Rep. 576, 1 (2015).

[8] G. S. Bali, F. Bruckmann, G. Endrodi, Z. Fodor, S. D. Katz, S. Krieg, A. Schafer, and K. K. Szabo, J. High Energy Phys. 02 (2012) 044.

[9] G. S. Bali, F. Bruckmann, G. Endrodi, Z. Fodor, S. D. Katz, S. Krieg, A. Schafer, and K. K. Szabo, Proc. Sci., LATTICE2011 (2011) 192 [arXiv:1111.5155].

[10] G. S. Bali, F. Bruckmann, G. Endrodi, Z. Fodor, S. D. Katz, and A. Schafer, Phys. Rev. D 86, 071502 (2012).

[11] M. D’Elia, Lect. Notes Phys. 871, 181 (2013).
[12] E. M. Ilgenfritz, M. Muller-Preussker, B. Petersson, and A. Schreiber, Phys. Rev. D 89, 054512 (2014).

[13] V. P. Gusynin, V. A. Miransky, and I. A. Shovkovy, Phys. Rev. Lett. 73, 3499 (1994); 76, 1005(E) (1996).

[14] V. P. Gusynin, V. A. Miransky, and I. A. Shovkovy, Phys. Lett. B 349, 477 (1995).

[15] V. P. Gusynin, V. A. Miransky, and I. A. Shovkovy, Phys. Rev. D 52, 4718 (1995).

[16] See, however, Ref. [17] and G. Aarts, L. Bongiovanni, E. Seiler, D. Sexty, and I.-O. Stamatescu, Eur. Phys. J. A 49, 89 (2013), and the references therein, for recent developments.

[17] G. Aarts, J. Phys. Conf. Ser. 706, 022004 (2016).

[18] J. O. Andersen, W. R. Naylor, and A. Tranberg, Rev. Mod. Phys. 88, 025001 (2016).

[19] J. M. Maldacena, Int. J. Theor. Phys. 38, 1113 (1999); Adv. Theor. Math. Phys. 2, 231 (1998).

[20] U. Gursoy, E. Kiritsis, and F. Nitti, J. High Energy Phys. 02 (2008) 019.

[21] U. Gursoy, E. Kiritsis, L. Mazzanti, G. Michalogiorgakis, and F. Nitti, Lect. Notes Phys. 828, 79 (2011).

[22] U. Gursoy and E. Kiritsis, J. High Energy Phys. 02 (2008) 032

[23] S. S. Gubser and A. Nellore, Phys. Rev. D 78, 086007 (2008).

[24] F. Bigazzi, R. Casero, A. L. Cotrone, E. Kiritsis, and A. Paredes, J. High Energy Phys. 10 (2005) 012.

[25] R. Casero, E. Kiritsis, and A. Paredes, Nucl. Phys. B787, 98 (2007).

[26] G. Veneziano, Nucl. Phys. B159, 213 (1979).

[27] M. Järvinen and E. Kiritsis, J. High Energy Phys. 03 (2012) 002.

[28] D. Arean, I. Iatrakis, M. Järvinen, and E. Kiritsis, J. High Energy Phys. 11 (2013) 068.

[29] T. Alho, M. Järvinen, K. Kajantie, E. Kiritsis, and K. Tuominen, J. High Energy Phys. 01 (2013) 093.

[30] T. Alho, M. Järvinen, K. Kajantie, E. Kiritsis, C. Rosen, and K. Tuominen, J. High Energy Phys. 04 (2014) 124; 02 (2015) 33.

[31] T. Drwenski, U. Gursoy, and I. Iatrakis, J. High Energy Phys. 12 (2016) 049.

[32] U. Gursoy, I. Iatrakis, M. Järvinen, and G. Nijs, J. High Energy Phys. 03 (2017) 053.

[33] F. Bruckmann, G. Endrodi, and T. G. Kovacs, J. High Energy Phys. 04 (2013) 112.

[34] N. Jokela, A. V. Ramallo, and D. Zoakos, J. High Energy Phys. 02 (2014) 021.

[35] D. Dudal, D. R. Granado, and T. G. Mertens, Phys. Rev. D 93, 125004 (2016).

[36] K. A. Mamo, J. High Energy Phys. 05 (2015) 121.

[37] N. Evans, C. Miller, and M. Scott, Phys. Rev. D 94, 074034 (2016).

[38] Since $V_{\mu}$ is dual to the baryon number, our $B$ has flavor independent couplings to matter and is identified as $e B_{\text {phys }}$ up to an $O(1)$ numerical coefficient, where $B_{\text {phys }}$ is the physical magnetic field.

[39] For $c \lesssim 1$ the phase diagram at $\mu=0$ clearly exhibits inverse magnetic catalysis and the chiral transition temperature decreases with $B$. The particular choice $c=0.4$ is optimal for qualitative agreement with the lattice results. 
[40] The phase diagram at zero $B$ differs slightly from that of Ref. [30] because the parameter $c$ of Ref. [32] was chosen differently.

[41] T. Alho, M. Järvinen, K. Kajantie, E. Kiritsis, and K. Tuominen, Phys. Rev. D 91, 055017 (2015).

[42] A. Armoni and K. Fukushima, Phys. Rev. D 89, 105001 (2014).

[43] G. S. Bali, F. Bruckmann, G. Endrödi, S. D. Katz, and A. Schäfer, J. High Energy Phys. 08 (2014) 177.

[44] P. M. Hohler and M. A. Stephanov, Phys. Rev. D 80, 066002 (2009).

[45] A. Cherman, T. D. Cohen, and A. Nellore, Phys. Rev. D 80, 066003 (2009).

[46] The curves in this figure end on the horizontal axis at a finite $\mu$, but, due to limited numerical accuracy, we show them only down to a finite cutoff temperature.

[47] A. Ballon-Bayona, M. Ihl, J. P. Shock, and D. Zoakos, J. High Energy Phys. 10 (2017) 038.
[48] O. Aharony, J. Sonnenschein, and S. Yankielowicz, Ann. Phys. (N.Y.) 322, 1420 (2007).

[49] D. Mateos, R. C. Myers, and R. M. Thomson, Phys. Rev. Lett. 97, 091601 (2006).

[50] S. Kobayashi, D. Mateos, S. Matsuura, R. C. Myers, and R. M. Thomson, J. High Energy Phys. 02 (2007) 016.

[51] F. Preis, A. Rebhan, and A. Schmitt, J. High Energy Phys. 03 (2011) 033.

[52] T. Sakai and S. Sugimoto, Prog. Theor. Phys. 113, 843 (2005).

[53] J. O. Andersen and A. Tranberg, J. High Energy Phys. 08 (2012) 002.

[54] Even though the suggested lattice calculation is viable in principle, it may be plagued with technical problems, e.g., big uncertainties in the analytic continuation from imaginary to real chemical potential and a small radius of convergence even at very small values of $\mu$.

[55] T. Alho, https://github.com/timoalho/VQCDThermo. 